\title{
Etudes chimiques et microbiologiques sur la maturation de fromages persillés. Influence de la flore microbienne selon différentes technologies*
}

\author{
par
G. OTTOGALLI, B. BIANCHI-SALVADORI, A. GALLI, L. CECCHI, P. RESMINI et G. VOLONTERIO \\ Istituto di Microbiologia agraria e di Industrie agrarie dell'Università di Milano, \\ Centro Sperimentale del Latte di Milano
}

Dans une précédente étude nous avons tracé le cadre de la maturation du Gorgonzola dans ses phénomènes chimiques et microbiologiques fondamentaux [1]. A cette occasion nous avions essayé de mettre en relation les modifications des principaux composants du fromage avec la microflore trouvée aux différents moments de la maturation ; au sein de la microflore présente, on avait reconnu l'importance prépondérante des Penicillium, des bactéries lactiques, ainsi que de la microflore de la croûte. Dans cette seconde phase de la recherche nous avons essayé de vérifier le cadre biochimique de la maturation et d'approfondir le rôle effectif tenu par les trois principaux groupements microbiens lorsqu'ils sont mis en mesure d'agir isolément l'un de l'autre. A cet effet, des formes expérimentales ont été prévues, et précisément :

1) Sans Penicillium.

2) Sans "starters".

3) Sans croûte.

Ces formes ont été examinées sous le profil chimique et microbiologique au cours de la maturation, et comparées avec du Gorgonzola témoin. Même en tenant compte du fait qu'avec la méthodologie adoptée, la succession typique de la microflore est troublée et par suite les fromages étudiés sont très différents du Gorgonzola, les résultats obtenus ont servi dans certains cas à confirmer et dans d'autres cas à élucider certaines des hypothèses qui avaient été formulées lors d'une précédente étude [1].

* Etude effectuée avec le concours du C.N.R. 


\section{MATERIEL ET METHODES}

\section{Matériel}

Les formes faisant l'objet de la recherche ont été obtenues par des traitements industriels effectués suivant la technologie normale [2], à partir de lait pasteurisé. Les modifications sont indiquées dans la description de chaque essai.

Formes sans croûte (SC) : les formes ont été réalisées suivant la technologie normale; on a essayé d'empêcher la formation de la croûte en enveloppant les formes avec une feuille de parafilm qui a été périodiquement enlevée pour faciliter la disparition du sérum.

Formes sans ferments lactiques thermophiles (SF) : les formes ont été produites suivant la technologie normale, mais sans ajouter de ferments lactiques.

Formes sans inoculation de Pénicillium (SP) : les formes ont été produites sans l'adjonction de spores de Penicillium au lait et sans effectuer le piquage à $15 \mathrm{j}$ de manière à obtenir des formes exemptes de persillé.

Témoin $(C)$ : pour chaque essai, à partir du même lait, des formes de contrôle ont été préparées en procédant selon la technologie standard.

\section{Méthodes}

\section{Méthodes Chimiques}

En ce qui concerne les déterminations chimiques traditionnelles relatives à la substance sèche, $\mathrm{pH}$, lactose, acidité, $\mathrm{N}$ total, $\mathrm{N}$ soluble à $\mathrm{pH} 4,6$ et $\mathrm{N}$ ammoniacal, on peut se reporter à la précédente étude [1]. N soluble dans l'eau : comme indiqué par Savini [3] ; $\mathrm{N}$ caséine soluble : calculée comme différence entre $\mathrm{N}$ non soluble dans l'eau et $\mathrm{N}$ soluble à $\mathrm{pH} 6$.

Extraction des caséines et séparations électrophorétiques : suivant les indications contenues dans une autre étude [4].

Séparation et extraction des amino-acides libres : avec analyseur automatique, comme indiqué dans une autre étude [4, 5]. Méthylcétones : extraction, conversion en 2,4-DNP-hydrazones et leur purification suivant la technique indiquée par Schwartz et al. $[6,7]$; analyse gaz chromatographique des hydrazones suivant Kallio [8]. Acides gras libres : suivant la technique indiquée par Kaderavek et Volonterio [9]. Amines volatiles : extraction, purification et leur conversion en hydrochlorhydrates en adoptant la technique indiquée par Tokita [10] ; transformation en benzoïl-dérivés et leur analyse gaz chromatographique comme indiqué par Gasco et Barrera [11]. 


\section{MÉthodes microbiologiques}

Les examens microbiologiques ont été effectués par prélèvement d'une abondante portion de la forme (200-300 g) ; le fromage prélevé a été additionné de sel tryptone comme diluant, à raison de 1:6 et soumis à homogénéisation en appareil Ultraturrax à $20000 \mathrm{t} / \mathrm{mn}$. Les déterminations suivantes ont été effectuées :

Bactéries non lactiques en gélose, incubation à $32^{\circ} \mathrm{C}$ pendant $48 \mathrm{~h}$ et à $20^{\circ} \mathrm{C}$ pendant $4 \mathrm{j}$.

Coliformes en désoxycholate lactose agar en double couche ; incubation à $32^{\circ} \mathrm{C}$ pendant $18-24 \mathrm{~h}$.

Bactéries lactiques : lactiques mésophiles en sérum gélosé de lait [12] et lait stérilisé, incubation à $10^{\circ} \mathrm{C}$ pendant $5-10 \mathrm{j}$.

Bactéries lactiques « totales » en sérum gélosé de lait et lait stérilisé, incubation à $30^{\circ} \mathrm{C}$ pendant 2-5 j.

Bactéries lactiques thermophiles en sérum gélosé de lait et lait stérilisé, incubation à $45^{\circ} \mathrm{C}$ pendant 2-5 j.

L'incubation des plaques de sérum à $30^{\circ} \mathrm{C}$ a été effectuée en atmosphère de $\mathrm{N}_{2}$ et $\mathrm{CO}_{2} 95+5$ p. 100 .

A partir des plaques d'agar sérum et de lait il a été procédé, avec examens microscopique et biochimique, à l'identification des espèces selon Sharpe et Fryer [13].

Leuconostoc en agar Mayeux [14], incubation à $22^{\circ} \mathrm{C}$ pendant 5-8 j. Identification des espèces selon Garvie [15].

Entérocoques en milieu liquide de Rothe, incubation à $37^{\circ} \mathrm{C}$ pendant $36-48 \mathrm{~h}$, et par suite confirmation en milieu de Litsky à $37^{\circ} \mathrm{C}$ pendant $36-48 \mathrm{~h}$ et différentiation par étalement sur milieu de Barnes à $37^{\circ} \mathrm{C}$ pendant $36-48 \mathrm{~h}$. Identification des espèces suivant Barnes [16].

Levures et moisissures en agar OGA [17], incubation à $20^{\circ} \mathrm{C}$ pendant $5 \mathrm{j}$.

\section{RESULTATS}

\section{Données technologiques et examen organoleptique}

Les données technologiques des traitements figurent dans le tableau 1. En ce qui concerne l'examen organoleptique, celui-ci a été effectué à la fin de la maturation pour chaque forme expérimentale par rapport au témoin respectif.

Formes de témoin : ces formes ont toutes montré un aspect normal de la pâte; des détachements réguliers avec un persillage uniforme, un goût et des odeurs caractéristiques sans défauts.

Forme sans croûte: pâte élastique peu protéolisée. Goût légèrement plus acidulé que le contrôle avec tendance an goût amer, à 


\section{TABLEAU 1}

Données technologiques des traitements de témoin C, sans croûte (SC), sans Penicillium (SP) et sans starters (SF)

\begin{tabular}{|c|c|c|}
\hline & $\mathrm{C}, \mathrm{SC}, \mathrm{SP}$ & SF \\
\hline $\begin{array}{l}\text { Quantité lait } \\
\text { Acidité du lait } \\
\text { Quantité starters } \\
\text { Acidité starters } \\
\mathrm{T}^{\circ} \text { pasteurisation } \\
\text { Acidité mélange lait + starters } \\
\text { Titre de la présure } \\
\text { Quantité de présure } \\
\mathrm{T}^{\circ} \text { emprésurage } \\
\text { Penicillium ajouté } \\
\text { Coagulation après } \\
\text { Premier brassage } \\
\text { Acidité sérum } \\
\text { Arrêt } \\
\text { Deuxième brassage } \\
\text { Acidité sérum } \\
\text { Durée traitement } \\
\text { Chauffage } 20^{\circ} \mathrm{C}-22^{\circ} \mathrm{C} \\
\text { Salage } \\
\mathrm{T}^{\circ} \text { locaux affinage } \\
\text { Humidité locaux affinage } \\
\text { Piquage (pas pour SP) } \\
\text { Durée affinage } \\
\text { Rendement après affinage }\end{array}$ & $\begin{array}{l}5001 \\
17 \text { Dornic } \\
2 \mathrm{p} .100 \\
128 \text { Dornic } \\
72^{\circ} \mathrm{C} \\
19 \text { Dornic } \\
10000 \\
40 \mathrm{ml} / 100 \mathrm{l} \\
29^{\circ} \mathrm{C} \\
\text { souche NO-C }(10 \mathrm{ml} / 100 \mathrm{l}) \\
17 \mathrm{mn} \\
\text { avec } \text { "spada " } \\
12 \text { Dornic } \\
18 \mathrm{mn} \\
\text { avec } \text { spannarola " } \\
13 \text { Dornic } \\
48 \mathrm{mn} \\
24 \mathrm{~h} \\
\text { à sec } \\
+5^{\circ} \mathrm{C} \\
90 \mathrm{p} .100 \\
15 \mathrm{gg} \\
60 \mathrm{gg} \\
\mathrm{kg} 12,3 \mathrm{p} .100\end{array}$ & $\begin{array}{l}5001 \\
17 \text { Dornic } \\
- \\
\overline{72} \\
72^{\circ} \mathrm{C} \\
17 \mathrm{Dornic} \\
10000 \\
40 \mathrm{ml} / 1001 \\
30^{\circ} \mathrm{C} \\
\text { souche NO-C }(10 \mathrm{ml} / 100 \mathrm{l}) \\
29 \mathrm{mn} \\
\text { avec } \text { "spada " } \\
11 \mathrm{Dornic} \\
28 \mathrm{mn} \\
\text { avec " spannarola " } \\
11 \mathrm{Dornic} \\
65 \mathrm{mn} \\
24 \mathrm{~h} \\
\text { à sec } \\
+5^{\circ} \mathrm{C} \\
90 \mathrm{p} .100 \\
15 \mathrm{gg} \\
60 \mathrm{gg} \\
11,8 \mathrm{p} .100\end{array}$ \\
\hline
\end{tabular}

attribuer probablement à un égouttement insuffisant. Penicillium assez bien développé et persillage normal. La croûte caractéristique ne s'est pas formée.

Forme sans "starters ": pâte élastique, crémeuse, bien égouttée, goût légèrement amer, ouverture accentuée, développement de Penicillium légèrement supérieur au témoin.

Forme sans "Penicillium »: pâte très compacte, sèche, crayeuse. Goût nettement plus acide que le contrôle ; dans l'ensemble l'aspect et le goût rappellent ceux du «Taleggio ».

\section{Résultats chimiques}

Dans le tableau 2 figurent les valeurs moyennes de $\mathrm{pH}$ et d'acidité titrable évaluées sur la masse du fromage. Le tableau 3 indique les valeurs constatées avec détermination stratigraphique. On note que le contrôle et le fromage SF se comportent de la 
TABLEAU 2

Analyses chimiques à 30 et $60 \mathrm{j}$ de maturation effectuées sur les échantillons : sans croûte (SC), sans starters (SF), sans Penicillium (SP) et témoin (C). Résultats pour $100 \mathrm{~g}$ de matière sèche

\begin{tabular}{|c|c|c|c|c|c|c|c|c|}
\hline \multirow[b]{3}{*}{$\begin{array}{l}\text { Matière sèche } \\
\text { pH moyen } \\
\text { Lactose } \\
\text { Acidité (p. } 100 \text { ac. lactique) } \\
\mathrm{N} \text { total } \\
\mathrm{N} \text { soluble } \mathrm{pH} 4,6 \\
\mathrm{~N} \text { soluble } \mathrm{H}_{2} \mathrm{O} \\
\mathrm{N} \text { ammoniacal } \\
\mathrm{N} \text { caséine solubilisé } \\
\mathrm{N} \text { caséine solubilisé } \\
\text { p. } 100 \mathrm{P}\end{array}$} & \multicolumn{2}{|c|}{$\mathrm{SC}$} & \multicolumn{2}{|c|}{ SF } & \multicolumn{2}{|c|}{ SP } & \multicolumn{2}{|c|}{ C } \\
\hline & $30 \mathrm{j}$ & $60 \mathrm{j}$ & $30 \mathrm{j}$ & $60 \mathrm{j}$ & $30 \mathrm{j}$ & $60 \mathrm{j}$ & $30 \mathrm{j}$ & $60 \mathrm{j}$ \\
\hline & $\begin{array}{r}49,92 \\
5,45 \\
1,70 \\
1,81 \\
5,62 \\
1,02 \\
1,49 \\
0,011 \\
0,47 \\
31,50\end{array}$ & $\begin{array}{c}50,00 \\
5,58 \\
0,00 \\
1,52 \\
5,88 \\
1,38 \\
1,93 \\
0,034 \\
0,45 \\
23,31\end{array}$ & $\begin{array}{l}50,42 \\
5,64 \\
1,34 \\
1,38 \\
5,80 \\
0,72 \\
1,88 \\
0,006 \\
1,16 \\
61,70\end{array}$ & $\begin{array}{l}50,61 \\
6,25 \\
0,00 \\
0,95 \\
5,86 \\
1,45 \\
2,57 \\
0,052 \\
1,12 \\
43,57\end{array}$ & $\begin{array}{l}52,64 \\
5,40 \\
1,09 \\
1,75 \\
5,93 \\
0,87 \\
1,35 \\
0,010 \\
0,48 \\
35,55\end{array}$ & $\begin{array}{l}54,19 \\
5,47 \\
0,00 \\
1,74 \\
5,94 \\
1,30 \\
1,50 \\
0,028 \\
0,20 \\
13,33\end{array}$ & $\begin{array}{l}51,51 \\
5,51 \\
0,96 \\
1,64 \\
5,96 \\
1,47 \\
1,98 \\
0,009 \\
0,51 \\
25,75\end{array}$ & $\begin{array}{r}51,94 \\
6,21 \\
0,00 \\
1,26 \\
5,91 \\
2,03 \\
2,78 \\
0,051 \\
0,75 \\
26,97\end{array}$ \\
\hline
\end{tabular}


TABLEAU 3

Détermination stratigraphique du $\mathrm{pH}$ avec électrode dans le fromage (valeurs moyennes) après $30 \mathrm{j}$ et $60 \mathrm{j}$ d'affinage.

Témoin (C), sans croûte (SC), sans Penicillium (SP), sans starters (SF)

\begin{tabular}{|c|c|c|c|c|c|c|c|c|}
\hline & \multicolumn{2}{|c|}{ C } & \multicolumn{2}{|c|}{ SC } & \multicolumn{2}{|c|}{ SP } & \multicolumn{2}{|c|}{ SF } \\
\hline & $30 \mathrm{j}$ & $60 \mathrm{j}$ & $30 \mathrm{j}$ & $60 \mathrm{j}$ & $30 \mathrm{j}$ & $60 \mathrm{j}$ & $30 \mathrm{j}$ & $60 \mathrm{j}$ \\
\hline Quelques cm de la croûte & 6,0 & $6,8 / 7$ & 5,0 & 6,5 & 5,5 & 5,8 & 6,0 & $6,8 / 7$ \\
\hline Centre & 5,0 & $6,2 / 7$ & 5,0 & $6,2 / 6,5$ & 5,2 & 5,4 & 5,0 & $6,2 / 7$ \\
\hline
\end{tabular}

même façon : alors que le $\mathrm{pH}$ au centre de la forme à $30 \mathrm{j}$ est à peu près celui du caillé, près de la croûte il s'avère moins acide à cause de l'action de la microflore superficielle ; à $60 \mathrm{j}$ le $\mathrm{pH}$ ne varie plus dans le sens stratigraphique, mais apparaît hétérogène par rapport à la distribution de la moisissure arrivant à des valeurs proches de la neutralité dans les parties persillées et des valeurs de $\mathrm{pH}$ inférieures dans les portions non persillées. La forme SC dans laquelle, en l'absence de la microflore de la croûte, l'action désacidifiante est due exclusivement au Penicillium, présente un $\mathrm{pH}$ plus bas que le contrôle et surtout sans variations entre la périphérie et le centre. Les formes SP sont celles qui restent plus acides : en effet dans ce cas l'action désacidifiante n'est exercée que par la microflore de la croûte.

Le lactose (tab. 2) est encore présent dans la forme de $30 \mathrm{j}$ de maturation, mais est totalement absent dans celles de $60 \mathrm{j}$. La valeur élevée constatée dans la forme SC à $30 \mathrm{j}$ doit être attribuée à l'égouttement plus faible de cette forme.

L'azote soluble (tab. 2) dans l'eau est plus élevé dans l'échantillon SF, avec une valeur similaire à celle du contrôle, tandis qu'il l'est moins dans SP et SC ; dans le premier cas il manque en effet l'action du Penicillium, et dans le deuxième celui de la microflore de la croûte.

La quantité de caséine soluble (qui peut être mise en rapport avec la consistance de la pâte, étant donné que ce qui contribue à rendre le fromage mou est la disgrégation du phospho-caséinate), est plus élevée dans SF (pâte crémeuse), tandis qu'elle est plus faible dans SP (pâte crayeuse). En analysant le rapport en pourcentage d'azote caséine solubilisée/azote soluble dans l'eau, nous constatons 
TABLEAU 4. - Composition en acides aminés libres du lyophilisé écrémé : mg/100 de lyophilisé écrémé

\begin{tabular}{|c|c|c|c|c|c|c|c|c|}
\hline & \multicolumn{2}{|c|}{ Sans croûte } & \multicolumn{2}{|c|}{ Sans starters } & \multicolumn{2}{|c|}{ Sans Penicillium } & \multicolumn{2}{|c|}{ Témoin } \\
\hline & $30 \mathrm{j}$ & $60 \mathrm{j}$ & $30 \mathrm{j}$ & $60 \mathrm{j}$ & $30 \mathrm{j}$ & $60 \mathrm{j}$ & $30 \mathrm{j}$ & $60 j$ \\
\hline $\begin{array}{l}\text { Acide aspartique } \\
\text { Thréonine } \\
\text { Sérine } \\
\text { Asparagine + Glutamine } \\
\text { Proline } \\
\text { Acide glutamique } \\
\text { Cystine } \\
\text { Glycine } \\
\text { Alanine } \\
\text { - } \mathrm{NH}_{2} \text { butyrique } \\
\text { Valine } \\
\text { Méthionine } \\
\text { Isoleucine } \\
\text { Leucine } \\
\text { Tyrosine } \\
\text { Phénylalanine } \\
\text { - NH }{ }_{2} \text { butyrique } \\
\text { Ornitine } \\
\mathrm{NH}_{3} \\
\text { Lysine } \\
\text { Histidine } \\
\text { Arginine }\end{array}$ & $\begin{array}{r}44,6 \\
40,1 \\
63,5 \\
244,8 \\
87,1 \\
30,9 \\
5,6 \\
28,7 \\
62,4 \\
\\
99,1 \\
43,4 \\
39,6 \\
250,7 \\
73,7 \\
160,2 \\
159,6 \\
58,8 \\
29,7 \\
110,2 \\
1,9 \\
81,7\end{array}$ & $\begin{array}{r}49,6 \\
52,6 \\
61,0 \\
299,0 \\
102,6 \\
109,4 \\
12,4 \\
36,9 \\
87,6 \\
+ \\
140,7 \\
58,9 \\
69,5 \\
367,5 \\
125,8 \\
239,9 \\
152,4 \\
87,4 \\
91,7 \\
208,1 \\
16,8 \\
133,5\end{array}$ & $\begin{array}{r}8,6 \\
0,8 \\
2,3 \\
8,0 \\
3,6 \\
22,9 \\
3,1 \\
2,2 \\
13,1 \\
7,8 \\
2,0 \\
3,8 \\
19,6 \\
6,2 \\
20,7 \\
3,1 \\
10,9 \\
16,5 \\
7,3 \\
1,5 \\
3,7\end{array}$ & $\begin{array}{r}36,0 \\
20,1 \\
28,5 \\
111,7 \\
62,0 \\
157,3 \\
5,7 \\
21,2 \\
54,6 \\
3,5 \\
64,5 \\
28,8 \\
38,7 \\
132,1 \\
44,6 \\
97,1 \\
14,8 \\
13,7 \\
140,6 \\
118,7 \\
39,4 \\
71,2\end{array}$ & $\begin{array}{r}27,3 \\
35,3 \\
49,1 \\
201,5 \\
67,6 \\
39,4 \\
4,4 \\
21,0 \\
50,5 \\
+ \\
73,0 \\
36,5 \\
31,6 \\
199,8 \\
55,3 \\
142,0 \\
120,1 \\
42,7 \\
26,7 \\
108,5 \\
6,5 \\
85,7\end{array}$ & $\begin{array}{r}45,9 \\
65,8 \\
61,8 \\
342,7 \\
126,2 \\
74,4 \\
13,0 \\
43,2 \\
65,7 \\
\\
137,4 \\
62,6 \\
68,6 \\
364,4 \\
95,0 \\
202,2 \\
214,7 \\
68,4 \\
76,2 \\
208,3 \\
28,0 \\
128,2\end{array}$ & $\begin{array}{r}26,3 \\
23,8 \\
33,8 \\
146,7 \\
59,0 \\
31,6 \\
5,0 \\
16,3 \\
50,5 \\
\\
75,9 \\
28,5 \\
31,1 \\
193,1 \\
59,2 \\
134,0 \\
94,9 \\
47,0 \\
25,2 \\
74,1 \\
4,2 \\
59,7\end{array}$ & $\begin{array}{r}78,6 \\
56,6 \\
62,6 \\
291,4 \\
172,0 \\
295,1 \\
15,3 \\
47,7 \\
92,7 \\
151,3 \\
67,3 \\
94,4 \\
321,9 \\
139,2 \\
212,4 \\
63,3 \\
89,5 \\
174,8 \\
302,2 \\
91,5 \\
95,9\end{array}$ \\
\hline Somme & 1716,3 & 2503,6 & 167,7 & 1304,8 & 1424,7 & 2492,7 & 1219,9 & 2915,6 \\
\hline
\end{tabular}


TABLEAU 5. - Composition en acides aminés libres du lyophilisé écrémé.

Les valeurs sont les mêmes du tableau 4, mais exprimés en p. 100 de partie peptidique

\begin{tabular}{|c|c|c|c|c|c|c|c|c|}
\hline & \multicolumn{2}{|c|}{ Sans croûte } & \multicolumn{2}{|c|}{ Sans starters } & \multicolumn{2}{|c|}{ Sans Penicillium } & \multicolumn{2}{|c|}{ Témoin } \\
\hline & $30 \mathrm{j}$ & $60 \mathrm{j}$ & $30 \mathrm{j}$ & $60 \mathrm{j}$ & $30 \mathrm{j}$ & $60 \mathrm{j}$ & $30 \mathrm{j}$ & $60 j$ \\
\hline $\begin{array}{l}\text { Acide aspartique } \\
\text { Thréonine } \\
\text { Sérine } \\
\text { Asparagine + Glutamine } \\
\text { Proline } \\
\text { Acide glutamique } \\
\text { Cystine } \\
\text { Glycine } \\
\text { Alanine } \\
\text { - } \mathrm{NH}_{2} \text { butyrique } \\
\text { Valine } \\
\text { Méthionine } \\
\text { Isoleucine } \\
\text { Leucine } \\
\text { Tyrosine } \\
\text { Phénilalanine } \\
\text { - NH }{ }_{2} \text { butyrique } \\
\text { Ornitine } \\
\text { NH }_{3} \\
\text { Lysine } \\
\text { Histidine } \\
\text { Arginine }\end{array}$ & $\begin{array}{r}2,60 \\
2,34 \\
3,70 \\
14,26 \\
5,07 \\
1,80 \\
0,33 \\
1,67 \\
3,63 \\
- \\
5,77 \\
2,53 \\
2,31 \\
14,61 \\
4,29 \\
9,33 \\
9,30 \\
3,42 \\
1,73 \\
6,42 \\
0,11 \\
4,76\end{array}$ & $\begin{array}{r}1,99 \\
2,10 \\
2,44 \\
11,94 \\
4,10 \\
4,37 \\
0,49 \\
1,47 \\
3,50 \\
+ \\
5,62 \\
2,35 \\
2,78 \\
14,68 \\
5,02 \\
9,58 \\
6,09 \\
3,49 \\
3,66 \\
8,31 \\
0,67 \\
5,33\end{array}$ & $\begin{array}{r}5,13 \\
0,48 \\
1,37 \\
4,77 \\
2,15 \\
13,65 \\
1,85 \\
1,31 \\
7,81 \\
- \\
4,65 \\
1,19 \\
2,26 \\
11,69 \\
3,70 \\
12,34 \\
1,85 \\
6,50 \\
9,84 \\
4,35 \\
0,89 \\
2,22\end{array}$ & $\begin{array}{r}2,76 \\
1,54 \\
2,18 \\
8,56 \\
4,75 \\
12,06 \\
0,44 \\
1,62 \\
4,18 \\
0,27 \\
4,94 \\
2,21 \\
2,96 \\
10,12 \\
3,42 \\
7,44 \\
1,13 \\
1,05 \\
10,77 \\
9,10 \\
3,02 \\
5,46\end{array}$ & $\begin{array}{r}1,95 \\
2,48 \\
3,45 \\
14,14 \\
4,74 \\
2,76 \\
0,31 \\
1,47 \\
3,54 \\
+ \\
5,12 \\
2,56 \\
2,22 \\
14,02 \\
3,88 \\
9,97 \\
8,43 \\
3,00 \\
1,87 \\
7,61 \\
0,46 \\
6,01\end{array}$ & $\begin{array}{r}1,84 \\
2,64 \\
2,48 \\
13,75 \\
5,06 \\
2,98 \\
0,52 \\
1,73 \\
2,64 \\
- \\
5,51 \\
2,51 \\
2,75 \\
14,62 \\
3,81 \\
8,11 \\
8,61 \\
2,74 \\
3,06 \\
8,36 \\
1,12 \\
5,14\end{array}$ & $\begin{array}{r}2,16 \\
1,95 \\
2,77 \\
12,02 \\
4,84 \\
2,59 \\
0,41 \\
1,34 \\
4,14 \\
- \\
6,22 \\
2,34 \\
2,55 \\
15,83 \\
4,85 \\
10,98 \\
7,78 \\
3,85 \\
2,06 \\
6,07 \\
0,34 \\
4,89\end{array}$ & $\begin{array}{r}2,69 \\
1,94 \\
2,15 \\
9,99 \\
5,90 \\
10,12 \\
0,52 \\
1,64 \\
3,18 \\
- \\
5,19 \\
2,31 \\
3,23 \\
11,04 \\
4,77 \\
7,29 \\
2,17 \\
30,7 \\
5,99 \\
10,37 \\
3,14 \\
3,29\end{array}$ \\
\hline
\end{tabular}


que dans les échantillons témoins il représente une valeur constante aussi bien à 30 j qu'à 60 j (26-27 p. 100).

Dans les autres échantillons ce pourcentage varie pendant l'affinage, ce qui peut être considéré comme un indice d'irrégularité dans l'évolution de la maturation. Les tableaux 4 et 5 indiquent les données relatives aux amino-acides libres.

On peut remarquer que, alors que dans les échantillons SC et $\mathrm{SP}$ les valeurs des amino-acides sont à peu près les mêmes que dans les contrôles, pour SF ces valeurs sont nettement inférieures, ce qu'il faut attribuer à une activité protéolytique insuffisante et de toute façon différente de la part des lactiques mésophiles. Si l'on examine chacun des amino-acides séparément, l'on obtient confirmation des observations figurant dans une précédente note [17] : dans le témoin (C) l'on a une augmentation constante d'acide glutamique et de lysine et une diminution d'acide $\gamma$-amino-butyrique. Pour l'acide glutamique il se vérifie une augmentation, dans le témoin, de 2,59 p. 100 à $30 \mathrm{j}$ à $10,12 \mathrm{p} .100$ à $60 \mathrm{j}$, tandis que dans l'échantillon SF les valeurs sont toujours élevées et dans l'échantillon SP toujours faibles. Dans le contrôle l'acide $\gamma$-amino-butyrique présente une forte diminution; dans l'échantillon SF les valeurs

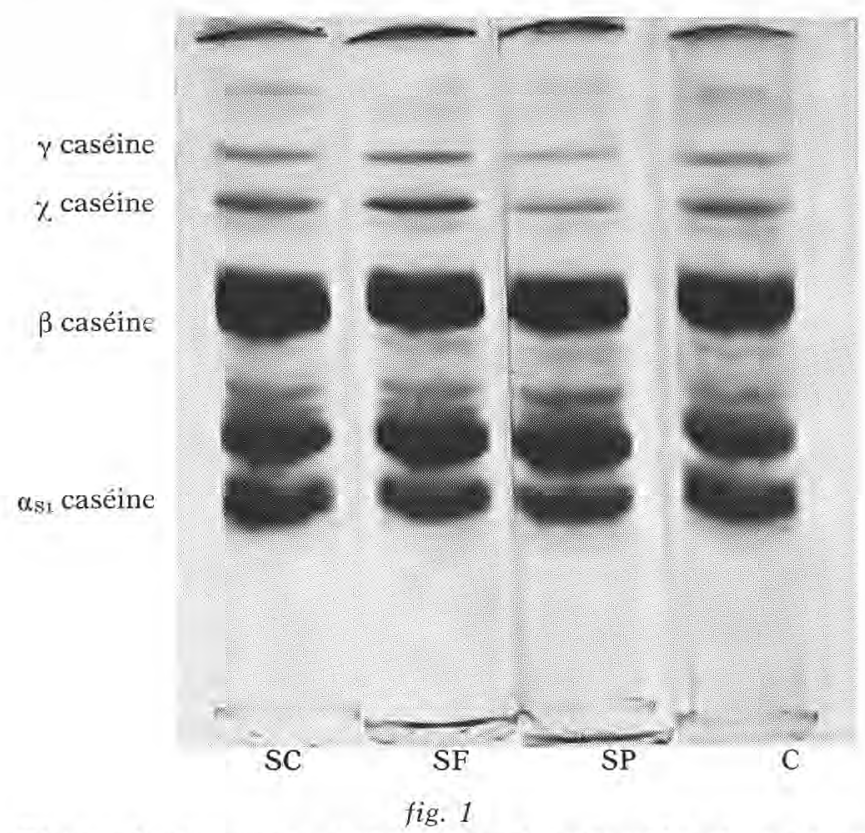

Electrophorégrammes (gélose de polyacrilamide) de caséines extraites de différents fromages après $30 \mathrm{j}$ d'affinage : sans croûte (SC), sans starters (SF), sans Penicillium (SP), témoin (C). 
restent basses aussi bien à $30 \mathrm{j}$ qu'à $60 \mathrm{j}$, et dans SP les valeurs sont élevées à $30 \mathrm{j}$ comme à $60 \mathrm{j}$. Il semble donc que les lactiques thermophyles soient en mesure d'accumuler des quantités considérables de cet amino-acide (ce qui s'accorde avec les données fournies par Salvadori [18] sur l'activité in vitro de L. bulgaricus). Par la suite, cet amino-acide est utilisé ultérieurement, mais seulement si le Penicillium est présent. Dans les figures 1-2 sont indiqués les tracés électrophorétiques des différents fromages; il est possible de cette façon d'observer le tracé du Gorgonzola à $60 \mathrm{j}$ dans lequel l'accumulation de produits de dégradation dans la zone de la $\gamma$-caséine est caractéristique. Comme cette dégradation manque dans le tracé de SP, tandis qu'elle est présente, bien que dans une plus faible mesure, dans SC et SF, on peut conclure que cette action est due au Pénicillium ; une preuve en est donnée par la comparaison de ce tracé avec celui du fromage Grana [19] où la protéolyse est due aux bactéries lactiques, avec accumulation de ces produits de dégradation dans la zone de la $\%$ mais non de la $\gamma$-caséine. Il faut noter que la $\beta$-caséine n'apparaît nettement attaquée que dans le contrôle, et par conséquent seulement dans le cas où sont présents tous les groupements microbiens. Enfin la caséine $\alpha$ s apparaît dégradée dans

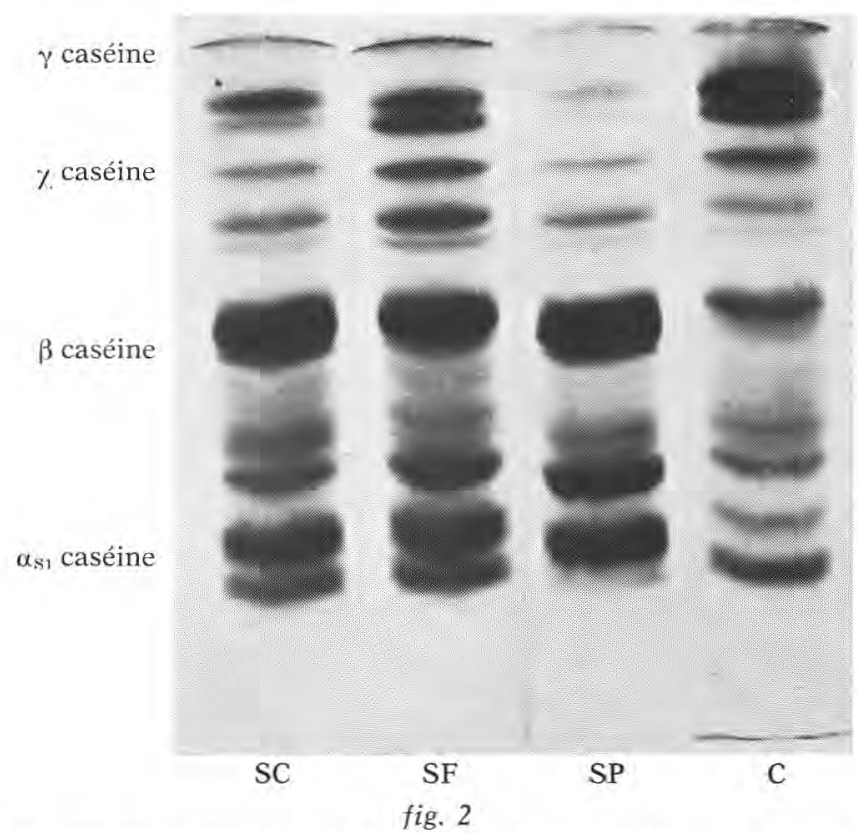

Electrophorégrammes (gélose de polyacrilamide) de caséines extraites de différents fromages après $60 \mathrm{j}$ d'affinage : sans croûte (SC), sans starters (SF), sans Penicillium (SP), témoin (C). 
une plus forte mesure, dans l'ordre par les moisissures, les lactiques, et la microflore de la croûte.

L'analyse du tableau 6 fait ressortir la présence de nombreuses amines aussi bien à $30 \mathrm{j}$ qu'à $60 \mathrm{j}$ de maturation, parmi lesquelles la di-iso-propyle et la di-iso-butyle-amine qui tendent à augmenter au cours de la maturation, et l'éthyle-amine qui au contraire subit une certaine diminution.

\section{TABLEAU 6}

Quantité approximative de quelques amines déterminées comme benzoyl-dérivés en 4 échantillons de fromage : sans croûte (SC), sans starters (SF), sans Penicillium (SP), témoin (C), après $30 \mathrm{j}$ et $60 \mathrm{j}$ d'affinage

\begin{tabular}{|c|c|c|c|c|c|c|c|c|}
\hline \multirow{2}{*}{ Amines } & \multicolumn{2}{|c|}{ SC } & \multicolumn{2}{|c|}{ SF } & \multicolumn{2}{|c|}{ SP } & \multicolumn{2}{|c|}{$\mathrm{C}$} \\
\hline & $30 \mathrm{j}$ & $60 \mathrm{j}$ & $30 \mathrm{j}$ & $60 j$ & $30 \mathrm{j}$ & $60 \mathrm{j}$ & $30 \mathrm{j}$ & $60 \mathrm{j}$ \\
\hline $\begin{array}{l}\text { Ethyl } \\
\text { Propyl } \\
\text { Diisopropyl } \\
\text { + isobutyl } \\
\text { Dipropyl } \\
\text { Diisobutyl } \\
\text { Hexyl }\end{array}$ & $\begin{array}{l}+++ \\
++ \\
+ \\
++ \\
++ \\
+ \\
+\end{array}$ & $\begin{array}{l}- \\
\operatorname{tr} \\
++ \\
+ \\
++ \\
+ \\
++\end{array}$ & $\begin{array}{c}+++ \\
++ \\
++ \\
+ \\
+ \\
+ \\
+\end{array}$ & $\begin{array}{c}- \\
++ \\
++ \\
+ \\
+ \\
+ \\
+\end{array}$ & $\begin{array}{c}- \\
+++ \\
+++ \\
- \\
- \\
+ \\
\text { tr }\end{array}$ & $\begin{array}{c}- \\
- \\
+++ \\
\operatorname{tr} \\
++ \\
++ \\
+\end{array}$ & $\begin{array}{c}++ \\
+ \\
++ \\
+ \\
++ \\
++ \\
+\end{array}$ & $\begin{array}{c}- \\
+ \\
+++ \\
+ \\
++ \\
++ \\
+\end{array}$ \\
\hline $\begin{array}{r}+=5 \mathrm{p} \cdot 100 \\
++=20 \mathrm{p} \cdot 100 \\
+++=40 \mathrm{p} \cdot 100\end{array}$ & & & & & & & & \\
\hline
\end{tabular}

Dans le tableau 7 on peut observer le comportement des acides gras volatils ; en termes généraux on note une diminution en pourcentage de l'acide acétique, et une augmentation de l'acide butyrique, en relation avec l'activité protéolytique, et surtout lipolytique des Penicillium et de la microflore de la croûte, ce qui est d'ailleurs confirmé par le fait que dans SC et surtout dans SP les valeurs de $C_{*}$ restent beaucoup plus basses que dans le contrôle. Le contenu en méthyle-cétones indiqué dans le tableau 8 met en relief l'importance en pourcentage de $\mathrm{C}_{\overline{5}}$ et $\mathrm{C}_{\overline{7}}$ au terme de la maturation ; dans le fromage entre $30 \mathrm{j}$ et $60 \mathrm{j}$ on note une diminution des méthylecétones $C_{3}$ et $C_{11}$ et une augmentation des $C_{3}, C_{5}$ et $C_{7}$.

\section{Résultats microbiologiques}

Les résultats microbiologiques figurent dans les tableaux 9, 10 et 11 avec les commentaires correspondants. 


\section{TABLEAU 7}

Quantité de quelques acides gras volatils libres en 4 échantillons de fromage : sans croûte (SC), sans starters (SF), sans Penicillium (SP), témoin (C), après $30 \mathrm{j}$ et $60 \mathrm{j}$ d'affinage (valeurs en p. 100)

\begin{tabular}{|c|c|c|c|c|c|c|c|c|}
\hline \multirow{2}{*}{ Acides gras } & \multicolumn{2}{|c|}{$\mathrm{SC}$} & \multicolumn{2}{|c|}{ SF } & \multicolumn{2}{|c|}{ SP } & \multicolumn{2}{|c|}{ C } \\
\hline & $30 \mathrm{j}$ & $60 \mathrm{j}$ & $30 \mathrm{j}$ & $60 \mathrm{j}$ & $30 \mathrm{j}$ & $60 \mathrm{j}$ & $30 \mathrm{j}$ & $60 \mathrm{j}$ \\
\hline $\begin{array}{l}\mathrm{C}_{2} \\
\mathrm{C}_{3} \\
\mathrm{C}_{4} \text { iso } \\
\mathrm{C}_{4} \\
\mathrm{C}_{5} \text { iso }\end{array}$ & $\begin{array}{r}89,97 \\
2,38 \\
1,03 \\
5,97 \\
0,63\end{array}$ & $\begin{array}{r}64,06 \\
2,68 \\
2,78 \\
17,84 \\
12,64\end{array}$ & $\begin{array}{r}85,48 \\
1,09 \\
2,66 \\
6,82 \\
3,95\end{array}$ & $\begin{array}{r}34,48 \\
2,03 \\
8,74 \\
41,30 \\
13,45\end{array}$ & $\begin{array}{r}83,77 \\
2,44 \\
2,65 \\
7,17 \\
3,96\end{array}$ & $\begin{array}{r}64,67 \\
1,57 \\
5,18 \\
14,75 \\
13,85\end{array}$ & $\begin{array}{r}74.80 \\
4,08 \\
4,28 \\
8,05 \\
8,78\end{array}$ & $\begin{array}{r}27,59 \\
1,70 \\
5,80 \\
51,54 \\
13,47\end{array}$ \\
\hline
\end{tabular}

TABLEAU 8

Quantité de quelques méthyl-cétones déterminés comme 2-4 DNP hydrazones en 4 échantillons de fromage : sans croûte (SC), sans starters (SF), sans Penicillium (SP), témoin (C), après $30 \mathrm{j}$ et $60 \mathrm{j}$ d'affinage (valeurs en p. 100)

\begin{tabular}{|c|c|c|c|c|c|c|c|c|}
\hline \multirow{2}{*}{ Méthyle-cétones } & \multicolumn{2}{|c|}{ SC } & \multicolumn{2}{|c|}{ SF } & \multicolumn{2}{|c|}{ SP } & \multicolumn{2}{|c|}{$\mathrm{C}$} \\
\hline & $30 \mathrm{j}$ & $60 j$ & $30 \mathrm{j}$ & $60 \mathrm{j}$ & $30 \mathrm{j}$ & $60 \mathrm{j}$ & $30 \mathrm{j}$ & $60 \mathrm{j}$ \\
\hline $\begin{array}{l}C_{3} \\
C_{5} \\
C_{7} \\
C_{3} \\
C_{11}\end{array}$ & $\begin{array}{r}4,01 \\
11,17 \\
20,04 \\
30,17 \\
30,61\end{array}$ & $\begin{array}{r}21,67 \\
31,02 \\
44,65 \\
1,27 \\
1,39\end{array}$ & $\begin{array}{r}8,36 \\
15,08 \\
19,74 \\
31,23 \\
25,50\end{array}$ & $\begin{array}{r}15,71 \\
38,35 \\
29,63 \\
10,95 \\
5,35\end{array}$ & $\begin{array}{r}24,63 \\
39,04 \\
31,38 \\
2,50 \\
2,44\end{array}$ & $\begin{array}{r}10,62 \\
37,86 \\
47,28 \\
2,04 \\
2,19\end{array}$ & $\begin{array}{r}7,64 \\
18,08 \\
22,64 \\
28,06 \\
23,57\end{array}$ & $\begin{array}{r}15,04 \\
39,04 \\
35,59 \\
6,58 \\
3,74\end{array}$ \\
\hline
\end{tabular}

Le fromage SC à la fin de la maturation s'est révélé bien persillé par des Penicillium roqueforti var. weidemannii. Les levures sont présentes à de faibles valeurs. La croûte caractéristique ne s'est pas développée, mais à certains endroits on a remarqué la présence d'une patine due à des bactéries psychotrophes gram - qui cependant se sont avérées métaboliquement inertes et par suite non en mesure d'influencer le biochimisme des couches inférieures. Il est apparu que la microflore lactique était constituée principalement de formes thermophiles de starter, et dans une quantité inférieure de mésophiles du lait (Str. lactis, L. casei, L. plantarum, L. brevis, 


\section{TABLEAU 9}

Nombre de micro-organismes/g de pâte au cours de la maturation.

Echantillons : sans croûte (SC) et témoin correspondant

\begin{tabular}{|c|c|c|c|c|c|c|c|c|}
\hline & & & \multicolumn{6}{|c|}{ Temps de prélèvement } \\
\hline \multicolumn{3}{|c|}{ Micro-organismes } & \multicolumn{2}{|c|}{ après salage } & \multicolumn{2}{|c|}{$30 \mathrm{j}$} & \multicolumn{2}{|c|}{$60 \mathrm{j}$} \\
\hline & & & $\mathrm{SC}$ & $\mathrm{C}$ & $\mathrm{SC}$ & $\mathrm{C}$ & $\mathrm{SC}$ & $\mathrm{C}$ \\
\hline \multicolumn{2}{|c|}{$\begin{array}{c}\text { Bactéries } \\
\text { non } \\
\text { lactiques }\end{array}$} & $\begin{array}{l}\text { totales } \\
\text { coliformes }\end{array}$ & $\begin{array}{c}9 \times 10^{7} \\
10^{5}\end{array}$ & $\begin{array}{c}9 \times 10^{7} \\
10^{5}\end{array}$ & $\begin{array}{c}4,2 \times 10^{7} \\
10^{5}\end{array}$ & $\begin{array}{l}2 \times 10^{7} \\
7 \times 10^{3}\end{array}$ & $\begin{array}{r}1,5 \times 10^{7} \\
2 \times 10^{3}\end{array}$ & $\begin{array}{r}1,5 \times 10^{7} \\
6 \times 10^{3}\end{array}$ \\
\hline \multirow{2}{*}{ 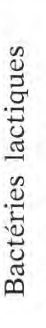 } & & $\begin{array}{l}\text { totales } \\
\text { thermophiles } \\
\text { mésophiles }\end{array}$ & $\begin{array}{l}1 \mathrm{X} 10^{9} \\
8 \mathrm{X} 10^{8} \\
3 \times 10^{7}\end{array}$ & $\begin{array}{l}1 \times 10^{9} \\
10^{9} \\
3 \times 10^{7}\end{array}$ & $\begin{array}{l}2 \times 10^{5} \\
8 \times 10^{7} \\
2,4 \times 10^{7}\end{array}$ & $\begin{array}{c}2 \times 10^{8} \\
3 \times 10^{8} \\
10^{7}\end{array}$ & $\begin{array}{c}1,5 \times 10^{8} \\
6 \times 10^{7} \\
10^{7}\end{array}$ & $\begin{array}{r}1,5 \times 10^{8} \\
6 \times 10^{7} \\
5 \times 10^{6}\end{array}$ \\
\hline & $\begin{array}{l}\text { Leuco- } \\
\text { nostoc } \\
\text { Entero- } \\
\text { coccus }\end{array}$ & $\begin{array}{l}\text { dextranicum } \\
\text { citrovorum } \\
\text { faecalis } \\
\text { durans }\end{array}$ & $\begin{array}{c}5 \times 10^{4} \\
4 \times 10^{8} \\
10^{3} \\
10^{5}\end{array}$ & $\begin{array}{c}5 \times 10^{4} \\
4 \times 10^{8} \\
\\
10^{3} \\
10^{5}\end{array}$ & $\begin{array}{c}4 \times 10^{6} \\
10^{7} \\
10^{3} \\
10^{5}\end{array}$ & $\begin{array}{c}4 \times 10^{6} \\
10^{7} \\
10^{4} \\
10^{4}\end{array}$ & $\begin{array}{c}4 X 10^{7} \\
4 X 10^{7} \\
\\
10^{5} \\
10^{5}\end{array}$ & $\begin{array}{c}4 \mathrm{X} 10^{7} \\
4 \mathrm{X} 10^{7} \\
\\
10^{5} \\
10^{5}\end{array}$ \\
\hline 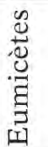 & & $\begin{array}{l}\text { Levures } \\
\text { Penicillium }\end{array}$ & $\begin{array}{l}1,6 \times 10^{6} \\
\text { prés. }\end{array}$ & $\begin{array}{l}1,6 \times 10^{6} \\
\text { prés. }\end{array}$ & $\begin{array}{l}9 \times 10^{\circ} \\
\text { prés. }\end{array}$ & $\begin{array}{l}5 \times 10^{6} \\
\text { prés. }\end{array}$ & $\begin{array}{l}5 \times 10^{6} \\
\text { prés. }\end{array}$ & $\begin{array}{l}9 \times 10^{6} \\
\text { prés. }\end{array}$ \\
\hline
\end{tabular}

L. buchneri et Leuconostoc spp. Ces derniers ayant été identifiés comme Leuc. dextranicum et Leuc. citrovorum, les deuxièmes en quantité plus forte. Il a été reconnu que les entérocoques appartenaient en quantité égale aux espèces faecalis et durans. Dans l'ensemble, à part la microflore superficielle, on ne note pas de différences substantielles entre les contrôles et les formes sans croûte, ce qui signifie que l'absence de cette microflore n'influence pas d'une manière particulière celle qui se trouve au-dessous.

Le fromage SF à la fin de la maturation s'est présenté bien persillé par des Penicillium roqueforti var. weidemannii.

La microflore de la croûte est celle caractéristique du Gorgonzola [1] : levures (Candida et Torulopsis) et bactéries (Micrococcus et Arthrobacter). 
TABLEAU 10

Nombre de micro-organismes/g de pâte.

Echantillons : sans «starters » $(\mathrm{SF})$ et témoin correspondant $(\mathrm{C})$

\begin{tabular}{|c|c|c|c|c|c|c|c|c|}
\hline \multirow{3}{*}{\multicolumn{3}{|c|}{ Micro-organismes }} & \multicolumn{6}{|c|}{ Temps de prélèvement } \\
\hline & & & \multicolumn{2}{|c|}{ Après salage } & \multicolumn{2}{|c|}{$30 \mathrm{j}$} & \multicolumn{2}{|c|}{$60 \mathrm{j}$} \\
\hline & & & SF & C & SF & C & SF & C \\
\hline \multirow{2}{*}{$\begin{array}{l}\text { Bactéries } \\
\text { non } \\
\text { lactiques }\end{array}$} & \multirow{2}{*}{\multicolumn{2}{|c|}{$\begin{array}{l}\text { totales } \\
\text { coliformes }\end{array}$}} & \multirow{2}{*}{$\begin{array}{r}3,5 \times 10^{7} \\
3 \times 10^{6}\end{array}$} & $4,5 \times 10^{7}$ & $6,6 \times 10^{7}$ & $8 \times 10^{6}$ & $1,2 \times 10^{8}$ & $2 \times 10^{\circ}$ \\
\hline & & & & $4 \times 10^{5}$ & $3 \times 10^{4}$ & $7 \times 10^{5}$ & $4 \times 10^{5}$ & $7 \times 10^{5}$ \\
\hline \multirow{3}{*}{$\begin{array}{l}\text { Bactéries } \\
\text { lactiques }\end{array}$} & \multicolumn{2}{|c|}{$\begin{array}{l}\text { totales } \\
\text { thermophiles } \\
\text { mésophiles }\end{array}$} & $\begin{array}{c}10^{9} \\
2 \times 10^{7} \\
5 \times 10^{8}\end{array}$ & $\begin{array}{r}3,2 \times 10^{8} \\
10^{9} \\
8 \times 10^{7}\end{array}$ & $\begin{array}{c}3 \times 10^{8} \\
10^{7} \\
10^{5}\end{array}$ & $\begin{array}{l}10^{8} \\
10^{8} \\
10^{7}\end{array}$ & $\begin{array}{c}2,5 \times 10^{8} \\
8 \times 10^{6} \\
10^{\circ}\end{array}$ & $\begin{array}{c}5 \times 10^{7} \\
10^{7} \\
10^{7}\end{array}$ \\
\hline & $\begin{array}{l}\text { Leuco- } \\
\text { nostoc }\end{array}$ & $\begin{array}{l}\text { dextranicum } \\
\text { citrovorum }\end{array}$ & $\begin{array}{l}2,4 \times 10^{6} \\
1,5 \times 10^{7}\end{array}$ & $\begin{array}{l}2,6 \times 10^{6} \\
1,5 \times 10^{7}\end{array}$ & $\begin{array}{r}8 \times 10^{6} \\
1,2 \times 10^{7}\end{array}$ & $\begin{array}{r}6 \times 10^{6} \\
1,3 \times 10^{7}\end{array}$ & $\begin{array}{l}8 \times 10^{6} \\
4 \times 10^{7}\end{array}$ & $\begin{array}{l}4 \times 10^{6} \\
5 \times 10^{7}\end{array}$ \\
\hline & $\begin{array}{l}\text { Entero- } \\
\text { coccus }\end{array}$ & $\begin{array}{l}\text { faecalis } \\
\text { durans }\end{array}$ & $\begin{array}{l}10^{3} \\
10^{3}\end{array}$ & $\begin{array}{l}10^{5} \\
10^{3}\end{array}$ & $\begin{array}{l}10^{5} \\
10^{5}\end{array}$ & $\begin{array}{l}10^{6} \\
10^{5}\end{array}$ & $\begin{array}{l}10^{6} \\
10^{5}\end{array}$ & $\begin{array}{l}10^{6} \\
10^{5}\end{array}$ \\
\hline \multirow{2}{*}{ Eumicètes } & \multicolumn{2}{|c|}{ Levures } & $5 \times 10^{5}$ & $3 \times 10^{s}$ & $6 \times 10^{5}$ & $8 \times 10^{\circ}$ & $7 \mathrm{X} 10^{7}$ & $7 \times 10^{7}$ \\
\hline & \multicolumn{2}{|c|}{ Penicillium } & prés. & prés. & prés. & prés. & prés. & prés. \\
\hline
\end{tabular}

Il est apparu que la microflore lactique était constituée principalement de formes mésophiles : Str. lactis, L. casei, L. buchneri et Leuconostoc, ces derniers étant identifiés comme Leuc. citrovorum et Leuc. dextranicum.

Les entérocoques sont subdivisés en deux espèces : Str. faecalis et Str. durans.

La différence entre les formes SF et celles de contrôle est due à la composition différente de la microflore lactique (en effet dans les formes SF elle est représentée principalement par des mésophiles, étant donné l'absence de "starters ») et au nombre plus élevé de coliformes, qui ressort d'ailleurs de l'ouverture plus abondante.

Le fromage SP s'est révélé exempt de Penicillium dans toutes les phases de la maturation, et particulièrement exempt de persillage à $60 \mathrm{j}$, à la différence du témoin. 
TABLEAU 11

Nombre de micro-organismes/g de pâte au cours de la maturation.

Echantillons sans Penicillium (SP) et témoin correspondant (C)

\begin{tabular}{|c|c|c|c|c|c|c|c|c|}
\hline & & & \multicolumn{6}{|c|}{ Temps de prélèvement } \\
\hline \multicolumn{3}{|c|}{ Micro-organismes } & \multicolumn{2}{|c|}{ Après salage } & \multicolumn{2}{|c|}{$30 \mathrm{j}$} & \multicolumn{2}{|c|}{$60 \mathrm{j}$} \\
\hline & & & SP & C & SP & $\mathrm{C}$ & SP & C \\
\hline \multicolumn{2}{|c|}{$\begin{array}{l}\text { Bactéries } \\
\text { non } \\
\text { lactiques }\end{array}$} & $\begin{array}{l}\text { totales } \\
\text { coliformes }\end{array}$ & $\begin{array}{r}30 \times 10^{7} \\
3 \times 10^{4}\end{array}$ & $\begin{array}{r}1,2 \times 10^{7} \\
3 \times 10^{+}\end{array}$ & $\begin{array}{c}2 \times 10^{\circ} \\
10^{\circ}\end{array}$ & $\begin{array}{c}2 \times 10^{\circ} \\
10^{2}\end{array}$ & $\begin{array}{c}3,2 \times 10^{7} \\
10^{3}\end{array}$ & $\begin{array}{r}4,5 \times 10^{7} \\
5 \times 10^{4}\end{array}$ \\
\hline \multirow{3}{*}{ 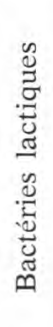 } & & $\begin{array}{l}\text { totales } \\
\text { thermophiles } \\
\text { mésophiles }\end{array}$ & $\begin{array}{c}10^{9} \\
6 \times 10^{8} \\
4,5 \times 10^{7}\end{array}$ & $\begin{array}{c}10^{9} \\
5 \times 10^{8} \\
4 \times 10^{7}\end{array}$ & $\begin{array}{c}10^{8} \\
8 \times 10^{7} \\
2,5 \times 10^{7}\end{array}$ & $\begin{array}{l}2 \times 10^{8} \\
7 \times 10^{7} \\
3 \times 10^{7}\end{array}$ & $\begin{array}{l}10^{\mathrm{s}} \\
10^{\mathrm{s}} \\
10^{\mathrm{s}}\end{array}$ & $\begin{array}{l}10^{5} \\
5 \times 10^{5} \\
5 \times 10^{7}\end{array}$ \\
\hline & $\begin{array}{l}\text { Leuco- } \\
\text { nostoc }\end{array}$ & $\begin{array}{l}\text { dextranicum } \\
\text { citrovorum }\end{array}$ & $\begin{array}{l}6 \times 10^{6} \\
6 \times 10^{7}\end{array}$ & $\begin{array}{l}4 \times 10^{6} \\
2 \times 10^{5}\end{array}$ & $\begin{array}{r}5 \times 10^{5} \\
1,5 \times 10^{5}\end{array}$ & $\begin{array}{r}1,5 \times 10^{7} \\
6 \times 10^{7}\end{array}$ & $\begin{array}{l}7 \times 10^{5} \\
4 \times 10^{7}\end{array}$ & $\begin{array}{l}4 \mathrm{X} 10^{6} \\
4 \mathrm{X} 10^{7}\end{array}$ \\
\hline & $\begin{array}{l}\text { Entero- } \\
\text { coccus }\end{array}$ & $\begin{array}{l}\text { faecalis } \\
\text { durans }\end{array}$ & $\begin{array}{l}10^{5} \\
10^{3}\end{array}$ & $\begin{array}{l}10^{5} \\
10^{3}\end{array}$ & $\begin{array}{l}10^{5} \\
10^{5}\end{array}$ & $\begin{array}{l}10^{5} \\
10^{5}\end{array}$ & $\begin{array}{l}10^{5} \\
10^{4}\end{array}$ & $\begin{array}{l}10^{5} \\
10^{5}\end{array}$ \\
\hline 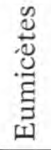 & & $\begin{array}{l}\text { Levures } \\
\text { Penicillium }\end{array}$ & $\begin{array}{l}6 \times 10^{5} \\
\text { abs. }\end{array}$ & $\begin{array}{c}2,2 \times 10^{6} \\
\text { abs. }\end{array}$ & $\begin{array}{l}7 \times 10^{5} \\
\text { abs. }\end{array}$ & $\begin{array}{c}8 \times 10^{\circ} \\
\text { abs. }\end{array}$ & $\begin{array}{c}3,5 \times 10^{6} \\
\text { abs. }\end{array}$ & $\begin{array}{l}2 \times 10^{7} \\
\text { abs. }\end{array}$ \\
\hline
\end{tabular}

La microflore de la croûte est celle caractéristique du Gorgonzola [1] ; levures (Candida et Torulopsis) et bactéries (Micrococcaceae et Arthrobacter). Il est apparu que la microflore lactique était constituée principalement de formes thermophiles du « starter » (L. bulgaricus et Str. thermophilus) et dans une mesure plus faible de mésophiles du lait (Str. lactis, L. casei, L. plantarum, L. brevis, L. buchneri et Leuconostoc, ces derniers ayant été identifiés comme Leuc. dextranicum et Leuc. citrovorum. Il a été reconnu que les entérocoques appartenaient en quantité égale aux espèces faecalis et durans. La différence substantielle entre la forme SP et le contrôle est constituée par l'absence de Penicillium. Aucune autre différence particulière à signaler. 


\section{CONCLUSIONS}

La microflore du Gorgonzola fabriqué suivant la technologie standard s'est révélée constituée par une riche association de bactéries lactiques et de Penicillium, ce qui confirme les indications contenues dans une précédente étude [1]. Les bactéries lactiques sont représentées : par les lactiques thermophiles des "starters " ( $L$. bulgaricus et Str. thermophilus) présentes dès le début à des valeurs de plusieurs centaines de millions/g, puis qui diminuent lentement; par les lactiques mésophiles qui se trouvent dans la cuve après la pasteurisation : celles-ci sont présentes au début en quantité toujours inférieure de plusieurs dizaines de millions à celle des thermophiles, bien qu'au cours de la maturation elles se maintiennent à des valeurs plus constantes, étant donné qu'aux températures d'affinage elles sont à même de survivre et, dans certains cas, de se multiplier. Elles se composent de : coques homofermentatifs (Str. Lactis), lactobacilles homofermentants ( $L$. casei et $L$. plantarum), coques hétérofermentants (Leuc. citrovorum et Leuc. dextranicum) et lactobacilles hétérofermentants ( $L$. brevis, L. buchneri). La microflore eumicétique est représentée fondamentalement par le Pénicillium roqueforti var. weidemannii et, en outre, par certaines levures de type Torula. Le Penicillium ajouté au lait sous forme de spores commence à sporuler et à devenir visible vers le $30^{\circ}$ jour, ce qui donne le caractéristique aspect persillé de la pâte.

La microflore de la croûte est représentée par des levures type Torula et des schizomycètes type Micrococcus et Arthrobacter qui confèrent la caractéristique couleur orangée.

D'intéressantes remarques sur l'importance réelle de chacun des groupements microbiens ont été mis en évidence par comparaison avec les formes à technologie modifiée. C'est ainsi que l'on a constaté que les formes sans croûte (SC) se distinguent du témoin (C) surtout par la plus forte acidité due à l'absence de l'action neutralisante de la microflore superficielle ; cette action, typique dans les fromages à maturation centripète, apparaît limitée dans le Gorgonzola aux couches superficielles et n'est pas en mesure d'influencer d'une manière considérable le biochimisme restant et les qualités organoleptiques. Le fromage obtenu sans les "starters " (SF) et laissé acidifier avec la microflore spontanée a permis un plus fort développement des coliformes, avec une ouverture évidente. Ceci provient de l'acidification inférieure dans les premières phases de la maturation; la solubilisation de la caséine a été satisfaisante mais la dégradation est dans son ensemble inférieure au contrôle, comme le prouve le tracé électrophorétique et le dégagement inférieur d'amino-acides : même sous le profil organoleptique, cette forme s'est révélée inférieure au témoin. Ces données confirment 
l'importance de la microflore des "starters " et des phénomènes d'interaction et complémentaires existant entre le Penicillium et les lactiques thermophiles [18]. Comme il ressort d'une précédente étude [1] l'influence du Penicillium est déterminante non seulement pour le persillage, mais aussi pour la structure de la pâte et pour le goût : la forme sans Penicillium a révélé des caractéristiques comparables à celles d'un fromage de type «Taleggio ». En présence de Penicillium, le tracé électrophorétique est caractéristique et suffit à rendre typique un fromage persillé par rapport à une maturation lactique : présence de fractions à basse vitesse électrophorétique dans la zone de la $\gamma$-caséine. L'action des moisissures apparaît encore déterminante vis-à-vis de l'activité lipolytique, comme le prouve la faible valeur de l'acide butyrique et des méthylcétones dans l'échantion non persillé.

En conclusion, il ressort que tous les groupements microbiens exercent un rôle important dans le processus de maturation, fonctionnant comme des anneaux s'encastrant par suite d'évolutions biochimiques successives, et qu'il n'est pas facile de varier la technologie sans compromettre la réussite d'un produit désormais standardisé et répondant à la demande du consommateur.

\section{Rés umé}

En vue d'améliorer les connaissances sur la chimie et la microbiologie du Gorgonzola, une étude a été effectuée sur des formes produites expérimentalement dans une fromagerie selon la technologie normale et parallèlement :

1) sans Penicillium,

2) sans "starters",

3) sans microflore de la croûte.

Il est apparu que la microflore du Gorgonzola est constituée par une riche association microbienne, se composant surtout de bactéries lactiques et de Penicillium.

Les formes sans croûte diffèrent du contrôle par une plus grande acidité due à l'absence de l'action neutralisante de la microflore superficielle (Micrococcus et Arthrobacter).

Les formes sans "starters ", qui permettent un plus fort développement de coliformes, ont en outre montré une dégradation des substances protéiques inférieure au contrôle, ce qui confirme l'importance des rapports d'interaction entre le Penicillium et les lactiques thermophiles inoculés. Enfin les formes sans Penicillium se sont révélées exemptes de persillé, plus acides et moins dégradées aussi bien du point de vue de la protéolyse que de la lipolyse. 


\section{S u $\mathrm{m} \mathrm{m}$ a r y}

In order to improve the knowledge of the chemistry and microbiology of Gorgonzola cheese, a research has been carried out on cheeses which were experimentally produced according to the normal technology and to parallel modified procedures : 1) without penicillium, 2) without starters, 3) without rind microflora.

The microflora of Gorgonzola has proved to consist of a rich microbial association, mainly represented by lactic acid bacteria and Penicillium.

The cheeses prepared without Penicillium proved to lack blue veining, to be more acid and to have undergone less proteolysis and lipolysis.

The cheeses produced without starters, besides allowing a stronger growth of coliform bacteria, showed weaker proteolysis than the control, which confirms the importance of the interaction between Penicillium and the thermophilic lactic acid bacteria.

The rindless cheeses differed from the control by a higher acidity due to the absence of the neutralizing effect of the rind microflora (Micrococcus and Arthrobacter).

\section{Bibliographie}

[1] Ottogalli (G.), Resmini (P.), Bianchi (B.), Galli (A.), Rondinini (G.), Salvadori (P.), Saracchi (S.), Volonterio (G.) (1971). - Ricerche sulla maturazione del Gorgonzola sotto il profilo chimico e microbiologico. Il Latte, 45, 776.

[2] Del Forno (G.) (1971). - Il formaggio Gorgonzola. Tipolitografia Atena, Roma.

[3] Savini (E.) (1946). - Il Latte. Hoepli, Milano.

[4] Resmini (P.), Volonterio (G.), Saracchi (S.), Annibaldi (S.) (1971). - Esperienze di fabbricazione di formaggio Parmigiano-Reggiano con enzima coagulante "Suparen ". Nota 2. Sci. Tecn. Lat. Cas., 22, 406.

[5] Resmini (P.), Saracchi (S.), Peri (C.) (1972). - Frazionamento e concentrazione dei costituenti azotati nel corso della ultrafiltrazione del latte. Atti SIPIA Congresso su "Applicazione dei processi per membrana nel settore lattiero casearie ", Milano.

[6] Schwartz (D. P.), Parks (O. W.) (1963). - Methyl Ketones in Roquefort cheese. J. Dairy Sci., 46, 1422.

[7] Schwartz (D. P.), Haller (H. S.), Mark Keeny (1963). - Direct quantitative isolation of mono carbonyl compounds from fats and oils. Anal Chem., 35, 2131 .

[8] Kallio (H.), Linko (R.), Kaitaranta (J.) (1972). - Gas-liquid chromatographic analisis of 2,4-dinitrophenyl hydrazones of carbonyl compounds. J. Chromatogr., 65, 355. 
[9] KaderaveK (G.), Volonterio (G.) (1966). - Ancora sull'analisi gas-cromatografica degli acidi grassi a catena corta. Nota 2. Riv. It. Sost. Grasse, 43, 9.

[10] Tокіта (F.), Hosono (A.) (1968). - Studies on and behaviour of amines produced by Brevibacterium linens. Milchw., 23 (11), 690.

[11] GASCo (L.), BARRERA (R.) (1972). - Use of derivates from gas-chrom. Identification of alcohols, primary and secondary amines and thiols in food aromas. Anal. Chim. Acta, 61, 253.

[12] Ottogalli (G.), Galli (A.) (1967). - Ricerche sul fermenti lattici della specie Streptococcus thermophilus. Nota 4. Reperimento e determinazione quantitativa nel latte e derivati. Ann. Micr., 17, 135.

[13] Sharpe (M. E.), Fryer (T. F.), Smith (D. C.) (1966). - Identification of lactic acid bacteria da Gibbs B. M. and Skinner F. A. "Identification methods for microbiologists " part. Academic Press London and New-York, 65.

[14] Majeux (J. V.), Sandine (W. E.), Elliker (P. R.) (1962). - A selective medium for detecting Leuconostoc organisms in mixed strain starter cultures. J. Dairy Sci., 45, 655.

[15] GaRvie (E. I.) (1960). - The genus Leuconostoc and its nomenclature. J. Dairy Res., 27, 283.

[16] Barnes (E. M.) (1959). - Differential and selective media for the faecal streptococci. J. Sci. Food. Agric., 12, 656.

[17] Mossel (D. A. A.), Bechet (J.), Lambion (R.) (1962). - La prévention des infections et des toxi-infections alimentaires. C.E.P.I.A. Bruxelles, 190.

[18] Bianchi (B.), Salvadori (B.) (1973). - Impiego razionale degli innesti nella produzione dei formaggi molli. Sci. Tecn. Lat. Cas., 24 (3), 161.

[19] Resmini (P.), Volonterio (G.), Saracchi (S.), Dieci (E.) (1973). - Studi sulla maturazione del formaggio Parmigiano-Reggiano ottenuto con latte di vaccine "Reggiane" e di vaccine "Frisone ". Sci. Tec. Lat. Cas. in corso di stampa. 